\title{
Narrativas sobre la Ruta de Atención Intersectorial de las Violencias Sexuales en Medellín
}

\author{
Narratives about the Intersectoral Care Route of Sexual \\ Violence in Medellin
}

\author{
Narrativas da Rota de Atenção Intersetorial das \\ Violências Sexuais em Medellín
}

\author{
Heidi Pulido Varón $n^{* *}$ \\ Recibido 24. 02. 2016 • Arbitrado 10. 03. 2016 • Aprobado 21. 04. 2016
}

\section{Resumen}

Medellín es pionera en procesos de atención a las violencias sexuales, pero ante la insistencia del problema en la realidad de la ciudad, se requiere visibilizar los avances y limitaciones de tales procesos. Objetivo: evaluar la eficacia y la efectividad de la Ruta de Atención Intersectorial de las Violencias Sexuales de la ciudad. Método: la investigación asumió un enfoque cualitativo de tipo narrativo. Abordó, a través de entrevistas semiestructuradas, a 22 funcionarios de las instituciones de justicia, salud y protección que integran dicha ruta y a organizaciones no gubernamentales, colectivos y mesas de trabajo sobre el tema. $R e$ sultados: se configuraron dos categorías de análisis: (a) una ruta que

\footnotetext{
El presente artículo se deriva de la investigación "Efectividad y Eficacia en la articulación de acciones institucionales para la atención de las víctimas de violencias sexuales a través de la Ruta de Atención Intersectorial de la ciudad de Medellín, año 2015". La misma articula los intereses investigativos del Comité Municipal para la prevención y atención de las violencias sexuales de Medellín, de la Alcaldía de Medellín (Secretaría de Inclusión social y familia), la Fundación Universitaria Luis Amigó, la Universidad de Antioquia y la Corporación Universitaria Adventista de Colombia.

** Psicóloga, Universidad de Antioquia; Magister en Desarrollo, Universidad Pontificia Bolivariana, Medellín; Docente Investigadora Fundación Universitaria Luis Amigó, Medellín, Colombia, heidi. pulidova@amigo.edu.co
} 
ayuda... pero hay que ayudarla, y (b) una ruta limitada, estrecha e inacabada. Conclusiones: se reconocen los avances y potencialidades de la ruta de atención, no obstante, existen falencias en términos de articulación y coherencia en las acciones institucionales.

Palabras clave: violencias sexuales, Ruta de Atención Intersectorial de las violencias Sexuales, Medellín.

\section{Abstract}

Medellín is a pioneer city in sexual violence care processes, but and due to the persistence of the problem in the reality of the city, is required to visible progress and limitations of such processes. $\mathrm{Ob}$ jective: To evaluate the efficacy and effectiveness of an Intersectoral Care Route for sexual violence in Medellín city. Method: research took a qualitative approach to narrative. The instruments applied were semi-structured interviews to 22 officials of the institutions of justice, health and protection that make up the route and non-governmental organizations, groups and workshops on the subject. Information Analysis system: Two categories of analysis were set: (a) a route that helps ... but you have to help it, and (b) a limited, narrow and incomplete route. Conclusions: the progress and potential of the route of care are recognized, however, there are limitations in terms of coordination and coherence in institutional actions.

Keywords: sexual violence, Intersectoral Care Route of sexual violence in Medellín.

\section{Resumo}

Medellín é pioneira em processos de atenção às violências sexuais. Porém, diante da persistência do problema na realidade da cidade, é preciso assinalar os avances e limitações desses processos. Objetivo: 
avaliar a eficácia e efetividade da rota de Atenção Intersetorial das violências sexuais de Medellín. Método: a pesquisa segue um enfoque qualitativo de tipo narrativo, aplicando entrevistas semiestruturadas a 22 pessoas que trabalham nas instituições de justiça, saúde e proteção que conformam a rota, ou que pertencem a organizações não governamentais, coletividades e mesas de trabalho. Processo de análise: configuram-se duas categorias: (a) uma rota que ajuda... mas precisa ser ajudada, e (b) uma rota limitada, estreita e incompleta. Conclusões: a rota tem avances e potencialidades, contudo existem dificuldades na articulação e coerência das ações institucionais.

Palavras chaves: violências sexuais, Rota de Atenção Intersetorial das violências sexuais, Medellín.

\section{Introducción}

Colombia, en coherencia con normativas internacionales (Convención de Belén do Pará, la Convención sobre la eliminación de toda forma de discriminación contra la mujer-CEDAW-, Declaración sobre la eliminación de la violencia contra la mujer, la Plataforma de acción de Beijing 95, la Declaración universal de los derechos humanos, entre otras), contempla a las violencias sexuales dentro de su sistema legal como un delito penal (Ley 360 de 1997, Ley 1257 de 2008, entre otras).

Sin embargo, las violencias sexuales constituyen un fenómeno complejo y plural que trasciende la perspectiva legal, instalándose desde esferas intimas y privadas hasta espacios públicos que expresan los profundos engranajes socio-culturales que perpetúan y legitiman las lógicas internas del fenómeno y conducen al silenciamiento de las víctimas. En efecto, más allá de las conceptualizaciones legales, las violencias sexuales expresan contenidos culturales y sociohistóricos que sostienen diferencias de género, formas de exclusión y prácticas patriarcales que operan ampliamente en la cotidianidad de las personas pese a los avances significativos en su reconocimiento e intervención. 
En el caso de Medellín, la ciudad se ha destacado por incorporar una perspectiva de derechos y género que ha permeado las iniciativas gubernamentales y privadas, procurando logros en términos de equidad, justicia y participación para poblaciones históricamente vulneradas como niños, jóvenes, mujeres, personas en condición de discapacidad y población LGTBI. La Política Pública de Prevención y Atención de las Violencias en la ciudad (Acuerdo o9 de 2006) logra concretar la formalización de un proceso coherente con la legislación nacional como la Ley 360 de 1997, la Ley 1236 del año 2008, la Ley 1257 de 2008, la Ley 1146 de 2007 y la Ley 1329 del año 2009.

Dicha política, es coordinada por el Comité Técnico de Atención y Prevención de las Violencias Sexuales, desde el cual se emprenden acciones de promoción, prevención, detección, atención, rehabilitación, vigilancia y control que permiten favorecer:

\begin{abstract}
El respeto a la diversidad sexual, la prevención, detección y eliminación del abuso y explotación sexual, la disminución de la prostitución, la atención integral de las personas afectadas por las violencias sexuales, la protección y restitución de los derechos vulnerados por las violencias sexuales, la vigilancia a la calidad de la atención, la rehabilitación de los agresores, el control y vigilancia de las personas y establecimientos que llevan a cabo, promueven o permiten las diversas violencias sexuales, especialmente el abuso sexual, la explotación sexual, la inducción a la prostitución y la trata de personas (Concejo de Medellín; 2006: 1).
\end{abstract}

Precisamente la ruta de atención a las violencias sexuales, resultado de un largo proceso liderado por este comité y foco del presente estudio, asume una perspectiva plural sobre las violencias sexuales, demandando la integralidad y la articulación institucional en los procesos de intervención de los sectores salud, justicia, protección y educación.

Sin embargo, pese a los mecanismos legales nacionales, la fuerza del tema de las violencias sexuales en la agenda pública de la ciudad y la existencia de una ruta intersectorial para su atención, aún se mantiene de forma significativa fenómenos como las violencias sexuales en espacios públicos y privados que bajo formas sutiles y directas evidencian 
la presencia del problema. La situación de la ciudad, no es ajena a las dinámicas departamentales y nacionales del problema. Según el grupo Centro de referencia nacional sobre violencia (2015) durante el 2014, Antioquia registró 2921 exámenes médico-legales por presunto delito sexual, de los cuales 2412 corresponden a mujeres y 409 a hombres. De la cifra departamental Medellín representa 1070 casos, de los cuales 907 son mujeres y 163 hombres (Grupo Centro de Referencia Nacional sobre Violencia, 2015). ${ }^{1}$ Aunque con una diferencia significativa en las cifras, el XII informe sobre la situación de violación de los derechos humanos de las mujeres en Medellín (Vamos mujer y la Corporación para la vida mujeres que crean, 2015)²ambién alerta sobre la agudización del problema en la ciudad al indicar que de las 1911 denuncias por presunto delito sexual, 1692 casos representan mujeres. La misma fuente recalca que el mayor número de casos de violencia sexual corresponde a edades entre los o y 17 años, siendo las más afectadas mujeres, mujeres jóvenes y niñas.

Estas cifras aunque alarmantes no alcanzan a revelar en sí mismas la magnitud de un problema, donde convergen asuntos complejos como los mitos que sustentan las diferencias de género, el conflicto urbano y el accionar de actores armados ilegales presentes en los territorios, el desconocimiento del marco de derecho y la debilidad institucional para responder de forma efectiva a las situaciones que involucran la violencia sexual. Tampoco develan las estructuras y dinámicas de poder que limitan las denuncias y sostienen trabas administrativas e ideológicas que limitan el acceso a los recursos institucionales y la adecuada gestión de los casos de violencia sexual en la ruta.

1 La información presentada por el Grupo Centro de Referencia Nacional sobre Violencia se condensa en el informe Forensis 2014 y se fundamentan en Fuentes como el Instituto Nacional de Medicina Legal y Ciencias Forenses / Grupo Centro de Referencia Nacional sobre Violencia / Sistema de Información para el Análisis de la Violencia y la Accidentalidad en Colombia / Sistema de Información de Clínica y Odontología Forense / Sistema de Información Nacional de Estadísticas Indirectas.

2 El informe de la situación de la violación de derechos de la mujer, es realizado anualmente por La Corporación para la vida Mujeres que Crean y la Corporación Vamos Mujer. Esta última ha señalado la frecuente diferencia entre las cifras manejadas por Medicina Legal y los Centros de Atención e Investigación Integral a las Víctimas de Delitos Sexuales (CAIVAS). 
Aunque el fenómeno de las violencias sexuales es amplio y puede ser abordado desde diversidad de aristas, todas válidas y necesarias, el presente documento se enfoca en presentar las narrativas que tienen los sujetos en condición de funcionarios de los diferentes sectores e instituciones sobre la ruta de atención intersectorial a las violencias sexuales. Tal propósito es importante, en tanto permite un primer acercamiento a los procesos institucionales de prestación del servicio de atención integral a las víctimas, desde la posición subjetiva de quienes los lideran y acompañan.

\section{Aproximación conceptual a las violencias sexuales y la ruta de atención intersectorial de la ciudad de Medellín}

La violencia, en todas sus manifestaciones, es un asunto netamente humano, una adquisición social desde la cual se direccionan las acciones, para obtener por la fuerza algo que voluntariamente es negado (Domenach, 1981). Existe en esta, una connotación de poder, de dominio y destrucción el otro, que permite a vertientes filosóficas, sociológicas y psicológicas defender la diferencia entre la violencia y la agresividad instintiva del mundo animal (Fromm, 1973). Sin profundizar en esta discusión que desborda los objetivos de este trabajo, se hace necesario indicar que se asume la violencia como un asunto de poder que trasciende elementos biológicos y bélicos de los estados, para comprenderse en esferas de lo privado y lo público, donde tienen lugar las interacciones humanas y donde las acciones violentas adquieren sentidos particulares según las estructuras culturales y momentos socio-históricos determinados.

Sin embargo, las condiciones sociopolíticas configuradoras del conflicto armado en Colombia han contribuido a sostener las situaciones que apuntan a diversificar las expresiones de la violencia, trasladando el impacto del conflicto político a las relaciones cotidianas de las comunidades urbanas y rurales. De modo que a esta violencia estructural-política, se anudan otras violencias como la intrafamiliar, la violencia de género, las violencias sexuales, entre otras. 
Específicamente las violencias sexuales representan un tipo de violencia de género, un delito, en las que se evidencian lógicas de dominio y poder que operan ligadas a la manipulación afectiva, el miedo, la cosificación del cuerpo, la amenaza, la devaluación en general, todas aquellas acciones que vulneran, anulan la dignidad y libertad de la persona, a través de la imposición de una conducta sexual no permitida en situaciones asimétricas. La Organización Mundial de la Salud (OMS), considera como violencia sexual:

Todo acto sexual, la tentativa de consumar un acto sexual, los comentarios o insinuaciones sexuales no deseados, o las acciones para comercializar o utilizar de cualquier otro modo la sexualidad de una persona mediante coacción por otra persona, independientemente de la relación de ésta con la víctima, en cualquier ámbito, incluidos el hogar y el lugar de trabajo (OMS, 2003:161).

En una línea similar la ONU indica que la violencia incluye una gama similar de actos, incluido el coito sexual intentado o forzado, contacto sexual no deseado, obligar a una mujer o a una niña o niño a participar en un acto sexual sin su consentimiento, comentarios sexuales no deseados, abuso sexual de menores, mutilación genital, acoso sexual, iniciación sexual forzada, prostitución forzada, la trata con fines sexuales, entre otros (Secretario General de la ONU, 2006).

En coherencia con la anterior conceptualización, el comité técnico coordinador de la ruta intersectorial para la atención y prevención de las violencias; las asume desde una perspectiva polimorfa y multicausal que incluye:

Desde el abuso sexual; la violencia sexual doméstica; el estupro, la violación sexual o acceso carnal violento; el incesto; la explotación sexual comercial; la trata de personas, la prostitución y su inducción; la pornografía; hasta el rechazo a la diversidad sexual; el soborno a la sexualidad, su disfrute y su reproducción y el impedimento de una libre opción por la planificación familiar. Pues todas son formas de atentar contra los derechos humanos sexuales y reproductivos" (Alcaldía de Medellín, 2013,6) 
Lo anterior permite señalar que las violencias sexuales poseen un amplio espectro, de modo que trascienden la esfera moral para situarse como una problemática de salud pública y de violación de derechos humanos, cuyo impacto rebasa las dimensiones individuales para marcar una impronta relacional en lo colectivo.

Como fenómeno multicausal, las violencias sexuales revelan la participación de elementos macrosociales y microsociales que convergen, para favorecer su origen y perpetuación en las relaciones sociales. Así, en lo macrosocial se pueden situar los modelos socioculturales que históricamente han sostenido la inequidad de género y las asimetrías de poder que fundamentan roles y mitos frente a hombres y mujeres. Igualmente, la agudización del conflicto interno y las precariedades de contextos marginados y excluidos, coadyuvan a mantener condiciones socioafectivas e institucionales (familia, escuela, iglesia, estado) que favorecen la instalación de las violencias sexuales, en detrimento de las poblaciones más vulneradas como mujeres y niños. Por su parte los ámbitos microsociales, articulados a las dinámicas antes expuestas, expresan características relacionales en hombres y mujeres, donde la violencia media las relaciones afectivas, promoviendo círculos repetitivos del fenómeno generación tras generación (Contreras, Bott, Guedes y Dartnall, 2010).

La perspectiva del modelo ecológico en la comprensión de las violencias, ha resultado útil a los enfoques de salud pública para explicar la etiología de las violencias sexuales, en tanto considera factores individuales, relacionales, comunitarios y sociales que se articulan de forma recíproca y compleja:

El modelo ecológico destaca las causas múltiples de la violencia y la interacción de los factores de riesgo que operan dentro de la familia y en los ámbitos social, cultural y económico más amplios. En un contexto de desarrollo, el modelo ecológico indica también el modo en que la violencia puede ser causada por diferentes factores en distintas etapas de la vida (Organización Panamericana de la Salud-OPS-, 2003,15).

En este mismo sentido, los enfoques feministas señalan un continuum (Sánchez, 2008; Barraza, 2009; Muñoz, 2011) para explicar que 
las violencias sexuales, como otras violencias de género, se configuran y expresan a lo largo del tiempo, en todo el ciclo vital y en épocas de paz y guerra. Paralelamente la noción de continuum, destaca la coexistencia de diferentes formas de violencia, de modo que la violencia sexual puede ser parte, por ejemplo, de la violencia intrafamiliar o la violencia del conflicto armado. Es decir que la naturaleza de los actos de violencia se asocian entre sí y a experiencias en ámbitos públicos y privados dando lugar a "un continuum de violencia que va tomando diversas formas y se reproduce en todos los escenarios sociales" (Barraza, 2009, 5).

Lo anterior constituye una afrenta contra los derechos fundamentales de las personas, que mantiene los estereotipos de roles asociados al sexo, vulnera la dignidad, la estima, autonomía y potencial de sus víctimas, menguando la calidad de vida de las comunidades y elevando los costos socio-económicos para los Estados. En efecto,

Aunque es difícil obtener cálculos precisos, los costos de la violencia se expresan en los miles de millones de dólares que cada año se gastan en asistencia sanitaria en todo el mundo, además de los miles de millones que los días laborables perdidos, las medidas para hacer cumplir las leyes y las inversiones malogradas por esta causa restan a la economía de cada país (Organización Panamericana de la Salud-OPS-, 2003,3).

Frente a este panorama, y en coherencia con las demandas de marcos internacionales, los dispositivos legislativos en Colombia han procurado la atención estatal sobre las violencias sexuales enfatizando en un fortalecimiento interno del estado para minimizar la vulneración de derechos que implican las distintas formas de violencia sexual. En este sentido, el marco jurídico permite reconocerlas y proveer un contexto de referencia que regula las acciones para prevenir, reconocer y sancionar las violencias sexuales, como también garantizar el restablecimiento de derechos de las víctimas. Se destacan en Colombia los siguientes marcos legislativos:

- La Ley 1236 del año 2008, por medio del cual se modifican algunos artículos del código penal relativos a delitos de abuso 
sexual de la Ley 599 del año 2000, "delitos contra la libertad, integridad y formación sexual".

- La ley 1257 de 2008 orientada a garantizar para todas las mujeres una vida libre de violencia, tanto en el ámbito público como en el privado, el goce efectivo de sus derechos, el acceso a recursos institucionales para su protección y atención, y la adopción de las políticas públicas necesarias.

- La Ley 1329 del año 2009 la cual modifica el título IV de la Ley 599 del año 2000 y se dictan otras disposiciones para contrarrestar la explotación sexual comercial de niños, niñas y adolescentes, el proxenetismo y la utilización o facilitación de medios de comunicación para ofrecer actividades sexuales con personas menores de 18 años de edad.

- La ley 1146 de 2007, enfocada en niños, niñas y adolescentes, exige la prevención de la violencia sexual y la garantía de procesos de prevención y atención integral en diferentes ámbitos.

- - Código Penal Colombiano, específicamente el título IV: Delitos contra la libertad, integridad y formación sexual.

Estas y otras disposiciones legales de orden nacional, son implementadas en los territorios locales a través de procesos y estancias articuladas que permiten organizar rutas para brindar de forma coherente, pertinente, coordinada y efectiva la prestación de un servicio orientado bajo el enfoque de derecho e integralidad que requieren las víctimas de violencias sexuales. La ruta que se implementa en la ciudad de Medellín, se nutre de la experiencia del Comité municipal de las violencias sexuales así como de los marcos legislativos como el código de infancia y adolescencia, Ley 1098/o6; la Ley 1146/julio 10 de 2007, Lineamientos de la política nacional de construcción de paz y convivencia familiar haz paz.

La ruta de atención intersectorial de las violencias sexuales en Medellín articula instituciones de los sectores de salud, Justicia y protección: Empresas Prestadoras de Servicios de Salud (EPS's), Fiscalía, Centros de Atención e Investigación Integral a las Víctimas de Delitos Sexuales (CAIVAS), Medicina Legal, comisarías de familia entre otras. 
Igualmente, convergen programas y proyectos de la Alcaldía como la Línea de atención 123 Social, la Línea de atención 123 Mujer y la Unidad de reacción inmediata (URI) (Alcaldía de Medellín, 2007).

\section{Metodología}

El interés por reconocer la efectividad y eficacia de la ruta de atención a las violencias sexuales en la ciudad de Medellín, llevó a elegir un diseño cualitativo de tipo narrativo para este estudio, que convocó a investigadores de las ciencias sociales de la Fundación Universitaria Luis Amigó, la Universidad de Antioquia y la Corporación Universitaria Adventista.

La idea de reconocer la ruta de las violencias sexuales más allá de indicadores estadísticos, implicó reconocer a los actores sociales implicados en el problema investigado, como portadores de un saber construido desde la subjetividad, la experiencia individual y colectiva en torno al fenómeno. Asimismo, este diseño permitió adoptar una perspectiva flexible e inductiva frente a la realidad estudiada.

Los informantes en esta investigación, fueron los funcionarios de las instituciones de justicia, salud y protección que participan en la ruta de atención de las violencias sexuales. También se abordaron miembros de organizaciones no gubernamentales, colectivos y mesas de trabajo que acompañan procesos relacionados con las violencias sexuales en la ciudad. La muestra se configuró de forma intencionada (Martínez, 2004), teniendo en cuenta a aquellos funcionarios que hacen parte del Comité Municipal de Atención y Prevención de las Violencias Sexuales

La información se recolectó a través de entrevistas semiestructuradas y la observación participante, desarrollada en espacios de reunión del Comité Municipal. En total se realizaron 22 entrevistas individuales y se participó de 9 reuniones del Comité Municipal, donde se obtuvieron notas de campo valiosas para nutrir el análisis de la información. Los datos obtenidos fueron sistematizados y sometidos a un proceso de análisis descriptivo y comprensivo del cual surgieron dos categorías: 
a) una ruta que ayuda...pero hay que ayudarla y b) una ruta limitada, estrecha e inacabada.

\section{Resultados}

Aunque la ruta condensa un esquema operativo que puede evaluarse de forma cuantificable, la investigación enfatizó en la necesidad de abordarla desde elementos percibidos y sentidos por los sujetos que tienen la condición de funcionarios. Se parte de la idea que sus elaboraciones discursivas frente al proceso, aunque traducen una posición personal marcada por factores como la experiencia en determinado cargo, la trayectoria en procesos relacionados con las violencias sexuales, la identificación con discursos institucionales, entre otros; también dan cuenta de evaluaciones apropiadas que permiten reconocer la efectividad y la eficacia de la ruta de atención a las violencias sexuales de Medellín.

A continuación se presentan algunos de los hallazgos relacionados con la percepción de los funcionarios que acompañan los procesos de la ruta de atención a las violencias sexuales desde diferentes sectores. El análisis presentado, se articula alrededor de dos categorías a) una ruta que ayuda...pero hay que ayudarla y b) una ruta limitada, estrecha e inacabada. De acuerdo a las exigencias éticas de la investigación, en los argumentos de los informantes, se protegió su identidad utilizando un código alfanumérico para la entrevistas.

\section{a) Una ruta que ayuda... pero hay que ayudarla}

En la información recolectada, aparece como constate la valoración positiva de los procesos de ciudad relacionados con las violencias sexuales. El trabajo continuado del Comité Municipal, la incorporación del enfoque de derecho, los programas de promoción y prevención así como la vigencia de políticas públicas son reconocidos como avances significativos, tal como se evidencia en los siguientes fragmentos:

Es de anotar entonces que según la política pública en violencias sexuales y su enfoque, desde el 2007, en Medellín, funciona la ruta de ciudad, en la que se ha 
invertido bastante pero que sobre todo ha permitido que en Medellín se forme la institucionalidad local (E09, Funcionario Salud).

Yo celebro que en Medellín existan las rutas de atención y que hoy las violencias sexuales estén legisladas, porque son mecanismos de atención y reparación de las personas afectadas, de lo que se trata es de pulirlas y optimizarlas y creo que también estamos en este proceso, más avanzados hoy que antes. El reto está en que todo se cumpla, pero definitivamente estaríamos peor si no estuvieran (E21, funcionario $\mathrm{ONG}$ ).

Lo anterior indica que la ciudad y los diferentes procesos sociopolíticos han permitido visibilizarse como modelo en gestión de políticas y estrategias para enfrentar la problemática de las violencias sexuales: "Esta es una ciudad líder en acción social y tenemos que reconocer las capacidades y fortalezas que como ciudad ya tenemos" (Eo6, funcionario alcaldía). La ruta no representa, solo un procedimiento operativo, sino que es el resultado de un arduo trabajo realizado por el colectivo ciudadano e institucional interesado en una intervención efectiva de las violencias sexuales, lo cual es valioso teniendo en cuenta la complejidad sociocultural que ha rodeado la historia de violencia en Medellín y específicamente el tema de las violencias sexuales.

Asimismo, se identifica que esta ruta ha permitido que la institucionalidad la apropie bajo la normatividad vigente lo cual resulta significativo porque permite mantener el tema en la agenda pública y con ello visibilizar la complejidad que encierra. A su vez, la ruta impacta al colectivo social porque los procesos que establece son claros y asequibles, tanto para el funcionario como para los usuarios; de modo que el conocimiento del proceso, facilita y promueve la movilización subjetiva y social, permitiendo acceder a recursos para la prevención de la problemática, la atención oportuna a las víctimas en diferentes ámbitos y la promoción de la justicia.

Aunado a lo anterior, aflora un sentido de satisfacción en el discurso de los funcionarios, puesto que las garantías de confidencialidad y respeto por la víctima son las banderas éticas de una atención que responde al marco de derechos fundamentales de la persona y a la transversalización de los discursos de equidad de género: 
Desde que la persona ingresa se le dan a conocer sus derechos, si es una persona adulta se la da a conocer sus derechos: que tiene derecho a un abogado que su información es reservada, que si no tiene abogado el Estado le provee uno, que tiene derecho a un hospital o si no la EPS o Sisben, si son niños de una vez las asume Bienestar familiar (E15, Funcionario Justicia).

No obstante, pese al optimismo con el que se reviste la ruta de atención a las violencias intersectoriales los funcionarios abordados no caen en juegos de idealización o devaluación de los procesos institucionales. Es decir, que poseen la capacidad de reconocer los alcances y limitaciones de la ruta, lo cual lejos de convertirse en un asunto negativo puede representar un punto de partida idóneo para procesos de mejoramiento.

\section{b) Una ruta limitada, estrecha e inacabada}

Es reiterado en el discurso de los funcionarios el carácter inacabado de la ruta, lo que significa que pese a representar un logro significativo en la ciudad aún tiene aspectos sensibles por mejorar y un largo camino para que se implemente de forma efectiva y coherente con las necesidades de los usuarios.

Este es un reto de ciudad, que todavía creo que la ruta tiene muchas dificultades en su activación por desconocimiento de algunos actores importantes, a pesar de todo lo que se ha hecho todavía falta mucho trabajo para que sea realmente efectiva como la necesitamos (E13, funcionario protección).

Entre las falencias que perciben los funcionarios en la ruta, se halla la incapacidad de una adecuada articulación interinstitucional e intersectorial "Las violencias sexuales implican en sí una atención intersectorial e integral. Esa es la gran falla a fortalecer en Medellín. No trabajamos articuladamente...” (Eo9, funcionario salud). Esta situación refleja, procesos poco coordinados e imposibilidades para lograr una corresponsabilidad alrededor de los casos:

En Medellín cada institución con empeño, trabaja desde su misión y lineamientos cumpliendo su encargo social. Pero no hay eficacia todavía en la atención de las violencias, no se puede 
medir un impacto, no hay criterios de impacto sobre la ruta, las instituciones más representativas tienen protocolos, pero institucionalizados, faltaría generar redes entre instituciones y así dar cuenta de una verdadera ruta de ciudad (E19, funcionario alcaldía de Medellín).

La ausencia de articulación repercute no solo en una inadecuada inversión de recursos sino en dinámicas institucionales que saturan a las poblaciones y agudizan la desconfianza institucional minimizando la efectividad de la ruta:

Es que las Secretarías de la Alcaldía de Medellín como Inclusión Social y Familia, Gobierno, Educación, Salud, Mujeres, hacen mucho y nada a la vez; porque, repiten acciones en los mismos sitios, los mismos conocidos vulnerables. Cuántas veces están a la vez en un mismo lugar varias secretarías compitiendo con sus proyectos a la misma población que manosean, mal enseñan y los cansan, con acciones puntuales y desagregadas. Por eso es que no se ven los resultados, eso es falta de efectividad, cierto? No muestran impacto, así se intervenga mucho y se gaste mucho (E11, funcionario $\mathrm{ONG}$ ).

Si bien, la ciudad ha alcanzado logros importantes y podido materializar una ruta sustentada en instrumentos legales e informes técnicos, el impacto real en la atención a las personas aún no logra otorgar una total satisfacción. Se halla un consenso en que esta situación no está determinada entonces por lineamientos y protocolos porque estos se hallan definidos y disponibles para las instituciones y en cambio las falencias radican en la falta de compromiso institucional que aun accediendo a la normatividad no ejecuta apropiadamente los procesos:

Mira en eso se han trabajado mucho con las IPS, pero yo pienso que dentro de la ruta si bien está muy bien planteados y es muy clara los 15 pasos que se tienen, muchas instituciones prestadoras de servicios de salud aun no le ven la importancia al personal de salud mental, específicamente donde yo le veo más fallas; porque toda persona que ingresa por una violencia sexual debe tener una atención médica y psicológica inmediata y normalmente nuestros registros del alto número de casos ocurre los fines de semana y no hay personal de psiquiatría o psicología disponible, como establece la ruta para que haga la evaluación (E17, funcionario alcaldía de Medellín). 
En este panorama, juegan un papel fundamental asuntos culturales muy complejos que limitan la efectividad y eficacia de la ruta y derivan acciones que revictimizan y vulneran la dignidad de las personas afectadas. Esta situación se asocia a la falta de sensibilidad frente a víctima, pues si bien es un asunto que se ha incrementado no logra romper del todo los estereotipos de género que culpabilizan o estigmatizan, y tampoco logra generar las suficientes garantías para vencer temores y miedos a activar la ruta a través de la denuncia:

[...] hay una revictimización, hay una mirada de que la mujer siempre es la culpable. Tanto curso que les dan y la cosa no cambia, no puedo decir que todos los funcionarios pero sí muchos, porque los conozco de cerca con sus actitudes [...] hay una mirada de funcionarios tanto de hombres como mujeres, porque nos encontramos con mujeres tan machistas, que no respetan cual es la realidad de la ciudad frente a la mirada de la mujer. Si no se mira a la mujer de igual a igual, no como objeto, por más investigaciones que se hagan, las cosas no pueden cambiar (E16, funcionario ONG).

Hay tres factores que afectan esa denuncia y el tránsito de la ruta: el primero es el miedo por todo el influjo al margen de la ley, bandas criminales que abusan de los muchachos y las muchachas que además de ejercer el dominio territorial también dominan el cuerpo [...] Es un obstáculo para la ruta porque el miedo no deja que la ruta comience. El otro asunto es la naturalización de las violencias sexuales, no se rechaza, no se ve como algo malo. [...] El otro, muy importante es que la ruta no siempre funciona, por cada caso que en la ruta no fluya genera una desesperanza de desestimulo impresionante, uno lo podría multiplicar por cien o por mil (E8, funcionario mesa).

Los discursos que están arraigados de forma profunda en el colectivo, logran operar de tal modo que legitiman las violencias sexuales y sostienen lógicas que inoculan el temor, el miedo y la desesperanza. Asociado a esto, la ruta no logra sostener la confianza institucional necesaria para que pueda resultar efectiva y eficiente: 
La ciudadanía no encuentra relación entre lo que ofrece la ruta y lo que da. A la gente además, le da miedo. Ay no, ellas sienten que se ponen más en riesgo si denuncian 0 activan la ruta, porque no se sienten protegidas por las instituciones que supuestamente las cobijan y dicen abiertamente que ellas están más seguras calladitas, muchas mujeres están seguras de que ellas mismas solucionan los problemas que les trae la violación, que en cambio quedan más expuestas si denuncian y divulgan su situación. Prefieren el problema mental interno que dar vía a un proceso, porque el miedo es muy grande. (E21, funcionario $\mathrm{ONG}$ ).

Esta situación se agudiza con problemas estructurales como la saturación del sistema judicial y la debilidad institucional para responder oportuna y efectivamente a las condiciones reales de las víctimas:

Los limites u obstáculos que tiene la fiscalía que es un problema de todas las instituciones, la defensoría, los juzgados, la logística, no dan abasto con casos de delitos sexuales, hay que partir por ampliar las instituciones y eso implica más fiscales más investigadores, mas policía judicial muchos más defensores y muchos más jueces (E20, funcionario justicia).

También es recurrente en las narrativas de los funcionarios al referirse a la ruta, asuntos relacionados con las condiciones contractuales. En primer lugar, las mínimas garantías para los funcionarios en términos de estabilidad laboral ocasionan alta movilidad del recurso humano que apoya las diferentes instituciones, repercutiendo en procesos interrumpidos o realizados con poca motivación.

La condición de los profesionales desde lo laboral como contratistas de la alcaldía y la ciudad es preocupante, porque somos agentes de cambio y los llamados a generar movilizaciones con la gente y si bien los presupuestos de los programas a nivel público se han incrementado, no siempre las retribuciones a nivel salarial van en consonancia con nuestro hacer, es decir es la retribución económica de los profesionales lo que siempre resulta más aporreado y entonces es como si hubiera una tendencia a procurar desarrollo a costa de un sector de la sociedad que somos los profesionales (E08, funcionario mesa).

Y en segundo lugar, también se identifica en el sentir de los funcionarios que los cargos de las diferentes instituciones aún están permeados 
por clientelismos que posicionan a personas con una formación mínima en el tema de las violencias sexuales, lo cual también afecta la fluidez de los procesos en la ruta, en tanto torna insuficientes los protocolos o lineamientos institucionales en los procesos de atención a las violencias sexuales. Los siguientes fragmentos dan cuenta de estas situaciones:

[...] no nos digamos mentiras muchas de estas administraciones son muy politizadas entonces permanentemente el 90\% de los funcionarios somos por prestación de servicios, somos absolutamente cambiantes y en la medida en que he no haya una continuidad en los procesos, estos funcionan mientras haya un funcionario que le apasione, que meta el cuerpo y dejan de funcionar cuando se cambian los profesionales. Ese es un problema serio y de fondo que al final los proyectos y programas dependen de las personas y si a estas las viven cambiando... por eso no se ve el progreso (E04, funcionario Alcaldía de Medellín).

Por otra parte, aunque la ruta es amplia porque involucra varios sectores, para algunos funcionarios revela su estrechez cuando se confronta con la imposibilidad de dar un lugar a organizaciones no gubernamentales y colectivos ciudadanos que llevan una trayectoria amplia frente al tema de las violencias sexuales:

Hay que involucrar a otros sectores e instituciones como parte de la ruta de ciudad, de esa forma será más efectivo el llamado a la corresponsabilidad y podríamos hablar de una ruta de ciudad que realmente recoja y comprometa a toda la institucionalidad (E07, funcionario Alcaldía de Medellín).

Igualmente frente a la visión de víctima y victimario, y frente a otros fenómenos próximos a la violencia sexual, la ruta resulta estrecha pues estos asuntos aún no se han definido lo suficiente en el marco de los procesos que establece. Primero porque se mantiene la visión de una víctima pasiva, minada por el agresor lo que supone dificultades para visibilizar su potencial durante el proceso de atención. Y en segundo lugar queda por fuera problemáticas como la explotación sexual de niños, niñas y adolescentes: 
Entre las cosas que se identificaron como críticas en el tema de la ruta es que aunque es muy amplia sentimos que el tema de explotación sexual no logra ser recogido por esta ruta. Primero porque esta ruta se activa con la denuncia y aquí el gran problema de la explotación sexual no hay denuncia. A diferencia del abuso, la explotación sexual que identifica un agresor específico, que generalmente es doméstico; la explotación sexual no hay denunciante...y no hay denunciante porque se da es de lo público, donde los agresores son múltiples, donde es muy difícil que los muchachos y muchachas se sientan víctimas y denuncien y donde la familia casi siempre ausente, y algunas veces es cómplice... entonces no hay quien denuncie, y por ese lado no hay una probabilidad de que se active la ruta (E6, funcionario mesa).

Por último, la estrechez de la ruta es percibida ante la nula o mínima consideración a un proceso de intervención con el victimario que trascienda o por lo menos se articule a la acción del sistema penitenciario:

\begin{abstract}
Si en Colombia las violencias sexuales son un delito, la justicia es un sector protagónico en su intervención, y tiene que responder tanto por la intervención a la víctima como al victimario. Yo siento que el tema del victimario, siempre se nos queda cojo. Las instituciones dicen es que nuestra especialidad es atender a la víctima, no trabajamos con victimarios. Y estos se le dejan supuestamente a Justicia, que lo único que hace es judicializar y desde un enfoque cultural, habría que rehabilitar, reintegrar, para que después éste pueda aportar a la reparación integral de la víctima [...] son muy pocos, mínimos los procesos terminados y los agresores judicializados por estos delitos y me atrevería a decir, que de rehabilitación a estos actores victimarios nada. (E14, funcionario Alcaldía de Medellín).
\end{abstract}

En conjunto estas situaciones derivan percepciones poco favorables en relación a la ruta y su funcionamiento así como frente al impacto social planeado que genera sentimientos de impotencia y desesperanza en los funcionarios, tal como lo ilustra la siguiente expresión:

Cuando uno acompaña a una usuaria a activar la ruta, con lo que se encuentra es con la tiranía de la falta de estructura... Eso es la ruta: No tiene una estructura... No hay planeación, planificación...Nada unificado" (E18, funcionario $\mathrm{ONG}$ ). 


\section{A modo de cierre}

La investigación centrada en las narrativas de los funcionarios, permite reconocer una versión cualitativa de los procesos de ciudad relacionados con la Ruta de Atención de las Violencias Sexuales. Es claro que el reconocimiento de avances no evita que los funcionarios identifiquen las limitaciones de un proceso, que en términos técnicos ha logrado concretar elementos importantes pero que no termina siendo plenamente efectivo al confrontarse con la realidad de la población afectada, las dinámicas institucionales y el arraigo cultural de mitos y creencias que legitiman las violencias sexuales.

Las tensiones originadas entre aquellos procesos planeados institucionalmente y las dinámicas que cotidianamente enfrentan los funcionarios de los diferentes sectores relacionados con la ruta, ponen de relieve la necesidad de trascender los planteamientos técnicos hacia aspectos operativos que puedan garantizar su total efectividad. Esta demanda implica no solo asuntos administrativos que permitan regulaciones y monitoreo al cumplimiento de indicadores; sino un arduo trabajo desde lo cultural, lo psicoafectivo y simbólico que incluya a los funcionarios y a los niveles administrativos en pro de un compromiso más real para contrarrestar la problemática.

Así, se hace necesario que la atención a los casos de las violencias sexuales se libere de la voluntad política pero refuerce los procesos de compromiso y sensibilización del recurso humano. Asociado a ello, también es importante que la ruta pueda gestionar mecanismos para enfrentar situaciones estructurales complejas como el clientelismo en los procesos de contratación de profesionales y la saturación de procesos que rebasan la oferta institucional.

Es también importante para garantizar la efectividad y eficacia de la ruta que esta se someta a una evaluación continuada y dinámica que involucre otros sectores, los colectivos u organizaciones ciudadanos y la sociedad civil. Esta evaluación debe revisar con sumo cuidado elementos que la investigación detectó como altamente sensibles a la 
discusión y el ajuste; por ejemplo la activación de la ruta a través de la denuncia, los sesgos que se mantienen sobre la víctima y el victimario e incluso situaciones como la explotación sexual y comercial de niños, niñas y adolescentes, que relacionándose con las violencias sexuales, no son reconocidos como tal.

Finalmente, es importante retomar la idea de una ruta abierta dispuesta a reelaborarse y retroalimentarse. Aunque la idea de articulación se expresa en el discurso de los funcionarios y en la misma estructura de la ruta, articularse implica un trabajo en red donde necesariamente un eslabón debe dar cuenta a los otros de sus procesos, en otras palabras no es posible pensar una ruta donde cada institución cumple a cabalidad sus funciones frente a las violencias sexuales pero desconoce la forma como opera otra. Adicional a este componente operativo, el componente afectivo y simbólico hacia la víctima también debe ser articulado, en términos de lenguajes o representaciones de esta y la disposición para el proceso; porque no basta cumplir los lineamientos o protocolos solo para no incurrir en omisiones que conlleven a una sanción legal.

\section{Referencias}

Alcaldía de Medellín. (2007). Ruta de atención intersectorial de las violencias sexuales en Medellín. Recuperado de http://files.cavmedellinsentencias.webnode. com.co/200000031ce4f9cf49a/ruta\%20violencias\%20sexuales.pdf.

Alcaldía de Medellín. (2013). Documento Técnico de categorización de la VIF y las VSX para Medellín y Antioquia. Recuperado de https://www.medellin.gov. co/irj/go/km/docs/wpccontent/Sites/Subportal\%20del\%20Ciudadano/Salud/Secciones/Programas\%20y\%20Proyectos/Documentos/2013/Salud\%20 Mental/Doc\%20\%20Clasificaci\%C3\%B3n\%20de\%2ola\%2oVIF\%20y\%20 las\%2oVSx.pdf. 
Asamblea General de las Naciones Unidas. (1948). Declaración Universal de los Derechos Humanos. Recuperado de http://www.acnur.org/t3/fileadmin/scripts/ doc.php?file=t3/fileadmin/Documentos/BDL/2001/o013.

Barraza, C. (2009). Continuum de la violencia: un desafío para las políticas públicas. Manzana de la discordia Vol. 4, No. 1: 19-25, Recuperado de http://www. bdigital.unal.edu.co/48321/1/continuumdelaviolencia.pdf.

Concejo de Medellín. (2006). Acuerdo og de 2006. Recuperado de https://www. medellin.gov.co/irj/go/km/docs/wpccontent/Sites/Subportal\%20del\%2oCiudadano/Equidad\%20de\%20G\%C3\%A9nero/Secciones/Normas/Documentos/2011/Acuerdo\%209\%20de\%202006.pdf.

Contreras, J., Bott, S., Guedes, A., Dartnall, E. (2010). Violencia sexual en Latinoamérica y el Caribe: análisis de datos secundarios. Iniciativa de Investigación sobre la Violencia Sexual. Recuperado de http://www.oas.org/dsp/documentos/Observatorio/violencia_sexual_la_y_caribe_2.pdf.

Naciones Unidas. (1979). Convención sobre la Eliminación de todas las formas de Discriminación contra la mujer -CEDAW-. Recuperado de http://www. un.org/womenwatch/daw/cedaw/cedaw19/ssa.pdf.

Convención de Belem do Pará. Convención Interamericana para Prevenir, Sancionar y Erradicar la Violencia contra la Mujer. (1994). Recuperado de http://www. acnur.org/t3/fileadmin/scripts/doc.php?file=t3/fileadmin/Documentos/ BDL/2001/0029.

Congreso de la Republica. (2008). Ley 1236 de 2008. Recuperado de http://www. oas.org/dil/esp/Ley_1236_de_2008_Colombia.pdf.

Congreso de la Republica. (1997). Ley 360 de 1997. Recuperado de http://www.equidad.scjn.gob.mx/biblioteca_virtual/Legislacion/OtrosPaises/Colombia/o1.pdf. Congreso de la Republica. (2008). Ley 1257 de 2008. Recuperado de http://www. sdmujer.gov.co/images/pdf/ley1257.pdf. 
Domenach, J. (1981). La violencia y sus causas. París: Editorial de la UNESCO.

Recuperado de http://www.psicosocial.net/grupo-accion-comunitaria/centro-de-documentacion-gac/fundamentos-y-teoria-de-una-psicologia-liberadora/psicologia-social/8o8-la-violencia-y-sus-causas/file.

Fromm, E. (1973). Anatomía de la destructividad humana. Recuperado de http:// www.ignaciodarnaude.com/textos_diversos/Fromm,Anatomia\%20de\%20 la\%2odestructividad\%2ohumana.pdf.

Grupo Centro de Referencia Nacional sobre Violencia. (2015.) Forensis 2014. Datos para la vida. Herramienta para la interpretación, intervención y prevención de lesiones de causa externa en Colombia. Bogotá, D. C: Imprenta Nacional Martínez, M. (2004). Ciencia y arte en la metodología cualitativa. México: Trillas. Muñoz, P. (2011.) Violencias intersectoriales. Debates feministas y Marcos Teóricos en el tema de la pobreza y violencia contra las Mujeres en Latinoamérica. Tegucigalpa: Central America Women’s Network (CAWN). Recuperado de http://www.cawn.org/assets/Violencias\%2oInterseccionales.pdf.

ONU Mujeres. (1995). Declaración política y documentos resultados de Beijing +5. Recuperado de http://www.unwomen.org/ /media/headquarters/attachments/sections/csw/bpa_s_final_web.pdf.

Organización Mundial de la Salud- OMS-. (2013). Hoja informativa, serie Comprender y abordar la violencia contra las mujeres. Washington, DC. Recuperado de http:// apps.who.int/iris/bitstream/10665/98821/1/WHO_RHR_12.37_spa.pdf.

Organización Panamericana de la Salud -OPS-. (2003). Informe mundial sobre la violencias y la salud. Washington D.C. Recuperado de http://apps.who.int/ iris/bitstream/10665/112670/1/9275315884_spa.pdf.

Sánchez, O. (2008). Las violencias contra las mujeres en una sociedad de guerra. Bogotá: Ruta Pacífica de las Mujeres Colombianas.

Secretario General de la ONU. (2006). Estudio a fondo sobre todas las formas de violencia contra la mujer. Nueva York, EEUU: Naciones Unidas. Recupera- 
do de http://daccess-dds-ny.un.org/doc/UNDOC/GEN/No6/419/77/PDF/ No641977.pdf?OpenElement.

Vamos Mujer y Corporación para la vida mujeres que crean. (2015). XIII Informe de la situación de violación de derechos humanos de las mujeres en Medellín 2014. Las mujeres en los medios: Estereotipo y cosificación. Medellín: Francisco Vélez 